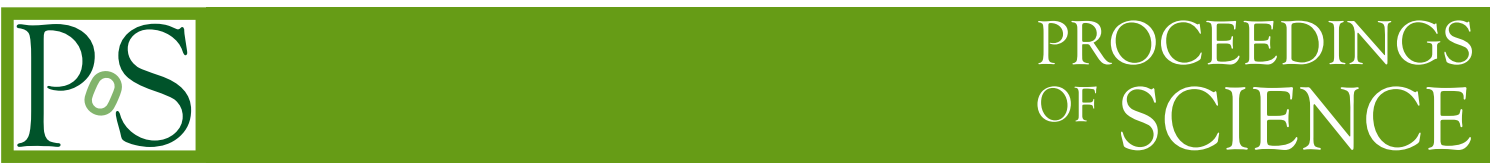

\title{
AGN life cycles - the low frequency story as told by LOFAR
}

\section{Aleksandar Shulevski*}

Kapteyn Astronomical Institute, Groningen University, The Netherlands Netherlands Institute for Radio Astronomy, The Netherlands

E-mail: shulevski@astro.rug.nl

\section{Raffaella Morganti}

Netherlands Institute for Radio Astronomy, The Netherlands

Kapteyn Astronomical Institute, Groningen University, The Netherlands

E-mail: morganti@astron.nl

Surveys of the low frequency radio sky now under way using the new LOFAR telescope are especially suited to search for extended AGN radio relics. These relics are remnants from previous cycles of AGN activity. As such, they are extremely interesting because through their studies we can determine the time AGNs spend in their on and off phases of activity. This constrains the total amount of energy delivered by AGNs into the interstellar / intergalactic environment, thus giving us a handle on estimating the impact AGNs make in galaxy evolution. Also, a detailed study of this AGN duty cycle may shed light on the accretion mechanisms responsible for the activity.

We present two cases of AGN radio relics, one discovered in data taken with the Westerbork Synthesis Radio Telescope (WSRT) and another imaged by the Low Frequency Array (LOFAR). We estimate the spectral index of the latter using our LOFAR image and radio survey data.

Nuclei of Seyfert galaxies and QSOs - Central engine $\mathcal{E}$ conditions of star formation November 6-8, 2012

Max-Planck-Institut für Radioastronomie (MPIfR), Bonn, Germany

\footnotetext{
*Speaker.
} 


\section{Introduction}

Evidence has been accumulating that active galactic nuclei (AGN) can have recurrent radioloud phases of activity (see for example Schoenmakers et al., 1999; Saikia \& Jamrozy, 2009). Thus, constraining the AGN duty cycle becomes very important since it's a gateway for understanding important physics. For example, the frequency of the activity can give us clues about the details of the accretion mechanism which works on very small scales around the AGN "engine" - a super massive black hole. In addition, the AGN cycle of activity determines the total energy returned to the interstellar and the intergalactic medium (ISM and IGM) in the radio loud phase of the life of a radio source. This "AGN feedback" affects the processes of galaxy formation and evolution.

Remnants of previous phases of AGN activity must be observed at lower frequencies, since as time passes the radiating particles (mostly electrons) lose energy and consequently their synchrotron spectrum steepens (e.g. Jaffe \& Perola, 1973; Kardashev, 1962; Murgia et al., 1999). In this proceeding we present two such cases. Section 2 discusses the radio relic which was discovered around the radio source B2 $0258+35$, hosted by the S0 galaxy NGC 1167 . This source was observed with the WSRT for purposes of H I study. Imaging the continuum data from this observation has uncovered the relic emission. Section 3 gives an overview of the LOFAR imaging of the B2 $0258+35$ radio source and its surroundings. This was a targeted observation attempting to detect the relic discussed in Section 2 at lower frequencies. In Section 3 we are looking more closely at another extended source in the obtained image - 4C 35.06 (associated with the core of the Abell cluster A 407). The LOFAR observations have uncovered an extension in one of the lobes. We present the spectral index study done using our LOFAR data. Throughout this writing, we define the spectral index $\alpha$ like: $S \propto v^{\alpha}$.

\section{2. $B 20258+35$}

B2 $0258+35$ was observed with the Westerbork array at $1.4 \mathrm{GHz}$. For details about the observing setup, data and reduction, see Shulevski et al. (2012). It is hosted by NGC 1167, a fascinating galaxy, containing also a huge $\mathrm{H}$ I disc with $\mathrm{M}_{H I}=1.5 \cdot 10^{10} \mathrm{M}_{\odot}$ and diameter of $160 \mathrm{kpc}$ (Struve et al., 2010a). A radio relic thought to originate from a previous stage of radio activity was discovered extending over $240 \mathrm{kpc}$ around the host galaxy. Interestingly, there is a young Compact Steep Spectrum (CSS) radio source active at a scale of $<1 \mathrm{kpc}$ signifying current activity of the AGN. The orientation of the CSS source matches that of the lobes of the larger relic (Figure 1).

Relics such as these are expected to have steep radio spectra, i.e. they appear more prominent at lower frequencies. This is a consequence of the fact that high frequency synchrotron radiation is radiated by charged particles with higher energy. As the time goes by (if there is no replenishment of these particles), only the low energy particles still radiate - at lower frequencies. Using observations over many different frequencies, we can estimate the age of the relic emission and thus determine the time at which the AGN activity ceased at that epoch (e.g. Murgia et al., 2011). We have observed B2 $0258+35$ at $325 \mathrm{MHz}$ using the WSRT for 12 hours, and we were unable to detect the relic. The non-detection sets a limit to the spectral index of $\alpha>-1.5$, which in turn may indicate that some sort of replenishing of the radiating particles is at work. Since at present we 

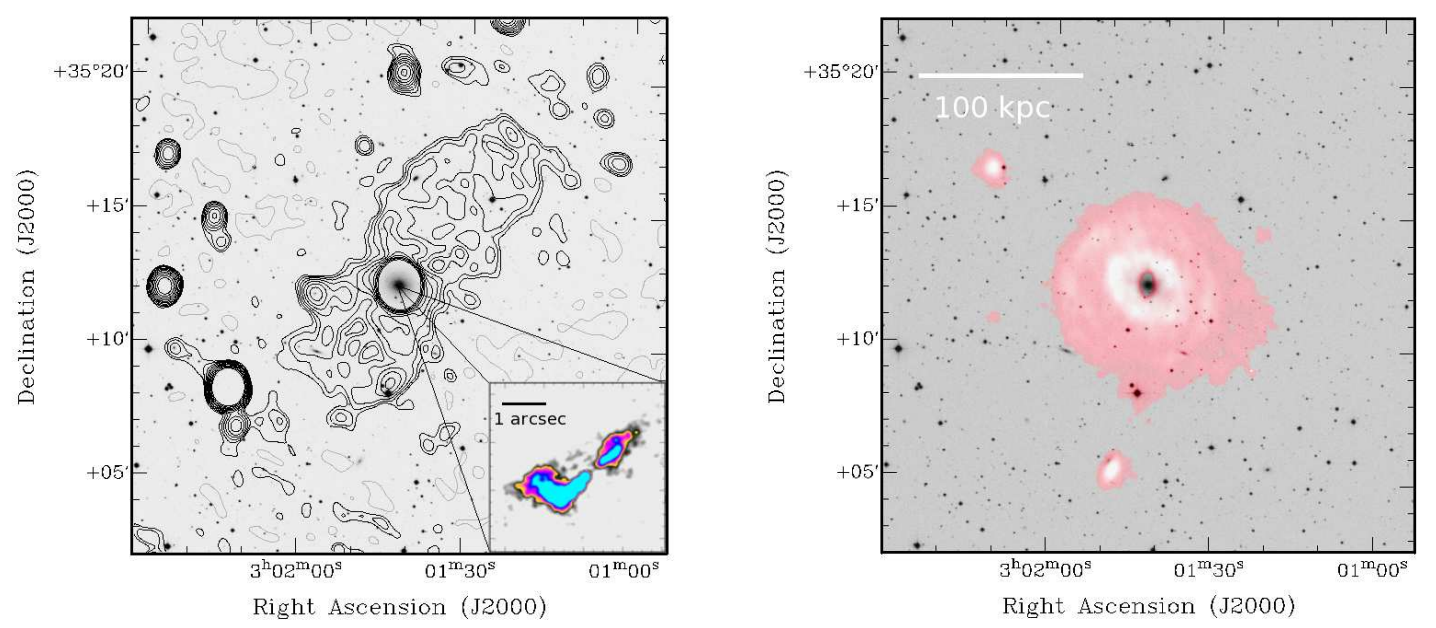

Figure 1: Left: continuum image of the diffuse emission around B2 $0258+35$. The synthesized beam is indicated with the ellipse at bottom left. The intensity ranges from $100 \mu J y(1 \sigma)$ to $2 \mathrm{mJy}$ $(20 \sigma)$. Right: $21-\mathrm{cm} \mathrm{H} \mathrm{I} \mathrm{total} \mathrm{intensity} \mathrm{image} \mathrm{showing} \mathrm{the} \mathrm{extent} \mathrm{of} \mathrm{the} \mathrm{H} \mathrm{I} \mathrm{disk} \mathrm{(red)} \mathrm{superposed}$ onto a DSS2 optical image of NGC 1167. Scale as indicated.

have flux density measurements of the relic only around $1.4 \mathrm{GHz}$, we can not employ synchrotron aging models to determine its age. We can get an approximate value for the age by using buoyancy arguments (Churazov et al., 2001). During the active AGN phase, the radio jets inflate lobes of plasma which push into the IGM. In this process, the mechanical energy of the expanding lobes does work against the pressure of the IGM, and the final size of the relic will depend on its radio power and the properties of the IGM. By measuring the size of the relic lobes, and making reasonable assumptions about the nature of the IGM, we can estimate the time it took for the relic to attain its present size. Using this argument, we have found its age to be around $8 \cdot 10^{7}$ years (Shulevski et al., 2012).

The source of accreting material for the AGN is also a mystery. The H I disk kinematics show no disturbances, indicating that the current episode of AGN activity is not a consequence of a recent merger or accretion of small galaxy companions. In addition, Emonts (2006) did not find evidence for a (merger related) star-burst in NGC 1167 at least in the past Gyr. H I distributed in a circumnuclear disk and an outflow in the core of NGC 1167 was observed by Struve et al. (2010a) against the radio continuum emission. We can speculate that this $\mathrm{HI}$ ring can be the source of the accreting gas. To get a clearer picture of the dynamics of the $\mathrm{H}$ in the core region, we have observed the core of NGC 1167 looking for H I in absorption against the core continuum using the European Very Large Baseline Interferometry Network (EVN). The data reduction is in progress at the time of this writing. In addition to this, gas supplied by cooling of the hot X-ray halo (Hardcastle, Evans \& Croston, 2007) or by stellar winds can be feeding the AGN. 


\section{3. $4 \mathrm{C} 35.06$}

The next logical step would be to study the relic in B2 0258+35 using the LOFAR telescope. So far, only a relatively shallow observation could be performed as part of the comissioning phase of observations with the array. B2 $0258+35$ and the area around it were observed at frequencies around $61 \mathrm{MHz}$ with $5 \mathrm{MHz}$ bandwidth in hopes of detecting the relic. In 8 hours of observing time, we have reached a noise level of around $30 \mathrm{mJy} \mathrm{beam}^{-1}$. The resulting image (centered on B2 $0258+35$ ) is given in Figure 2. The relic was not detected during this attempt, but we are confident that this will be possible in the near future. We estimate that the LOFAR observation puts a limit on the spectral index of the relic of $\alpha_{61}^{1400}>-1.3$ (consistent with what we have estimated in Section 2 above).

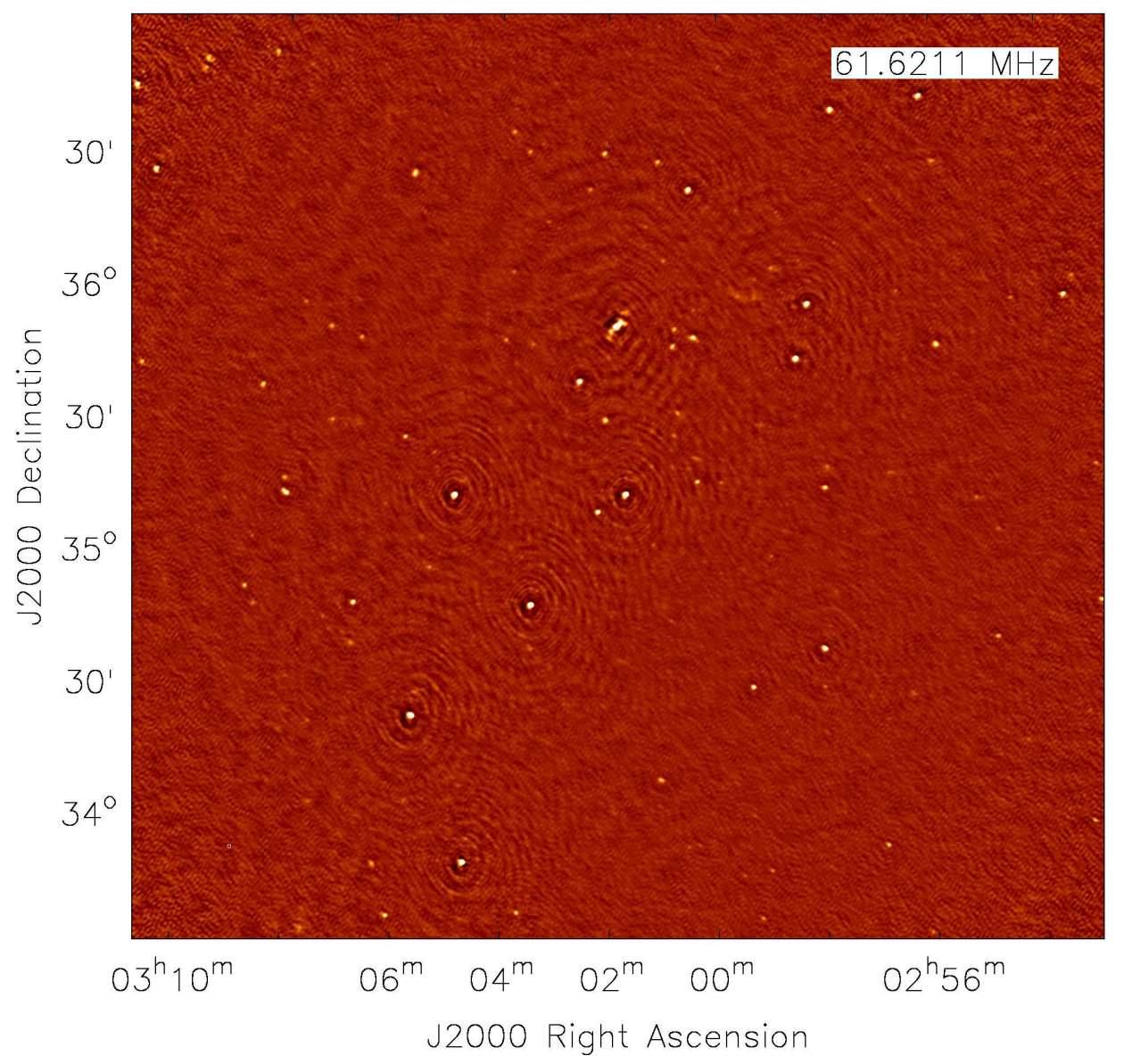

Figure 2: LOFAR intensity image ( 3 x 3 degrees, beam size: 48 " x 43") of the field centered on B2 $0258+35$. The radio source $4 \mathrm{C} 35.06$ is about half a degree to the north. The image is saturated to better show fainter structures.

We have detected 4 extended radio sources (NVSS J0307+3529, NVSS J0252+3556, NVSS $\mathrm{J} 0300+3520$, 4C 35.06), the most prominent of which is $4 \mathrm{C} 35.06$, located about half a degree 

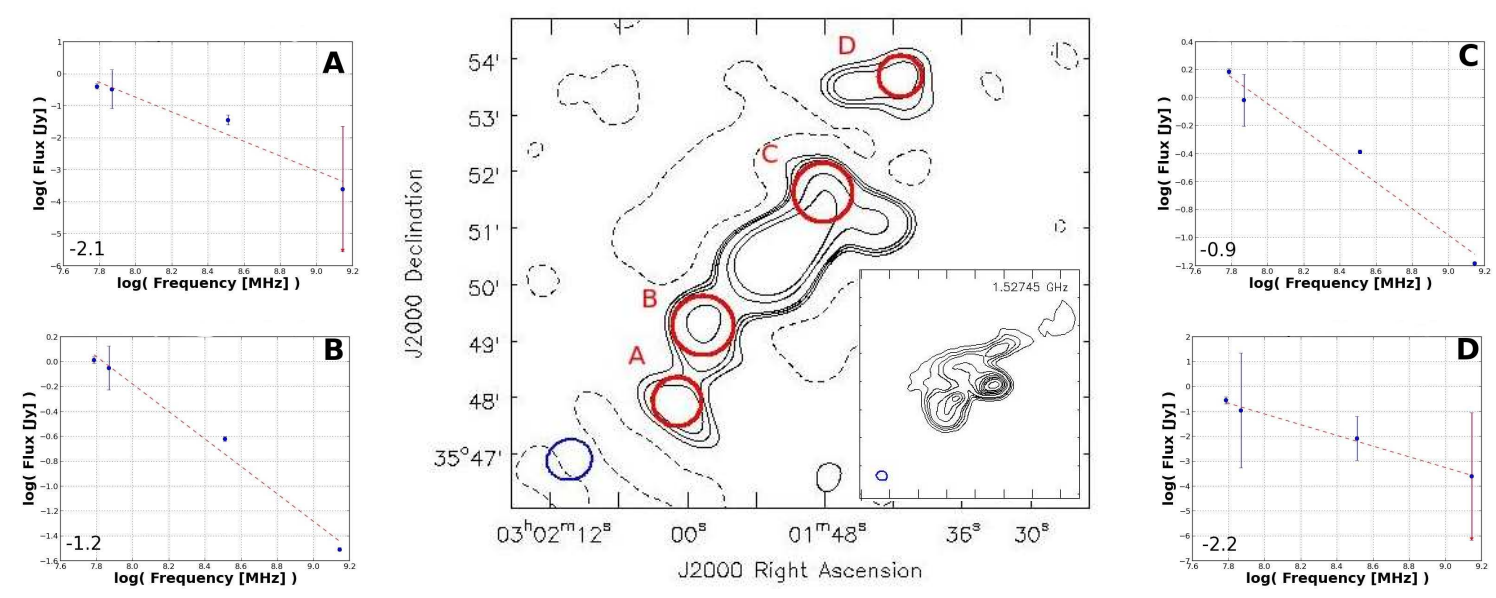

Figure 3: LOFAR contour image of 4C 35.06. Contours are drawn at: $(-6,6,9,12,30,51) \sigma$. Negative contour levels are dashed. The spectral index measurement regions are marked in red, and the synthesized beam is outlined in blue. The inset shows a $1.55 \mathrm{GHz}$ VLA contour map of the central region, and the accompanying plots give the spectral index measured for each of the marked regions.

north of the target. 4C 35.06 is an intriguing radio source, identified with the multi-core cD galaxy UGC 2489 which is part of the core of the galaxy cluster Abell 407. The source can be divided in at least four aligned components apart from the core (labeled A, B, C and D - with the aim of studying their spectral index), as can be seen in Figure 3.

We can expect that the regions of the source farthest from the center should be remnants of older AGN outbursts of activity. To quantify this, we have studied the details of the change in spectral index along the lobes of 4C 35.06 using our LOFAR image, and images from the VLSS, WENSS and NVSS radio surveys. Four distinct regions were measured, encompassing inner and outer parts of the nortwestern and southeastern lobes (C, D and A, B respectively). Care has been taken to define the measurement regions such as they fall in areas where flux in the LOFAR, VLSS, WENSS and NVSS images is above $3 \sigma$ respectively. Exception from this rule are the regions A and $\mathrm{D}$ where there is no detection in NVSS, so the measured fluxes are only upper limits. It's worth to note that using LOFAR we have confirmed the detection of an outermost northwestern lobe (identified with region D) which was only hinted at in VLSS data. This suggests the possibility of it having a very steep spectral index, signifying the oldest observable signature of AGN activity in this source. The derived spectral indices for each region are given in the lower left corner of the plots in Figure 3.

It can be seen that the spectral index of the lobes increases as one moves away from the central region, which is an expected trend if the outermost lobes trace the oldest episode of activity of the AGN. Further, the LOFAR detection (identified with region D) has the steepest spectral index.

Note that the spectral index was determined by a simple power-law fit to the data, which does not reflect a possible presence of a spectral curvature. In the future, we will have better frequency cov- 
erage (including the High Band Antenna (HBA) range of LOFAR frequencies spanning $120 \mathrm{MHz}$ - $240 \mathrm{MHz}$ ), allowing us to do this procedure in more detail. Identifying break(s) in the frequency spectrum can provide us with constraints on the age of the emitting plasma as well as give us limits to the duration of the active and inactive periods of the AGN.

Studying steep spectrum sources is also important because they include young CSS sources (in some cases their spectra can turn over at lower frequencies). These sources are thought to be in the beginning stages of life leading to a full fledged radio galaxy, and if their spectra show a turnover, the turnover frequency is correlated with their size. With this in mind, we have explored the properties of unresolved sources in the imaged region of the sky around B2 $0258+35$. Specifically, to determine their spectral shapes and whether we can detect steep spectrum sources. To do this, we have taken all $5 \sigma$ detections in the LOFAR image and matched them to sources in the WENSS and NVSS catalogs. The total number of sources matched was 90. Then, we've determined their $\alpha_{61}^{325}$ (LOFAR / WENSS), $\alpha_{325}^{1400}$ (WENSS / NVSS) and $\alpha_{61}^{1400}$ (LOFAR / NVSS) spectral indices. Plotting the WENSS-NVSS vs. the LOFAR-WENSS spectral index as shown in Figure 4 allows us to investigate the curvature of the spectra. We can see one source with a turnover in its spectrum. LOFAR surveys will help us uncover more of these sources and we can use the the turnover frequency to examine the nature of the medium surrounding the source (distinguishing between free-free and synchrotron self absorption).

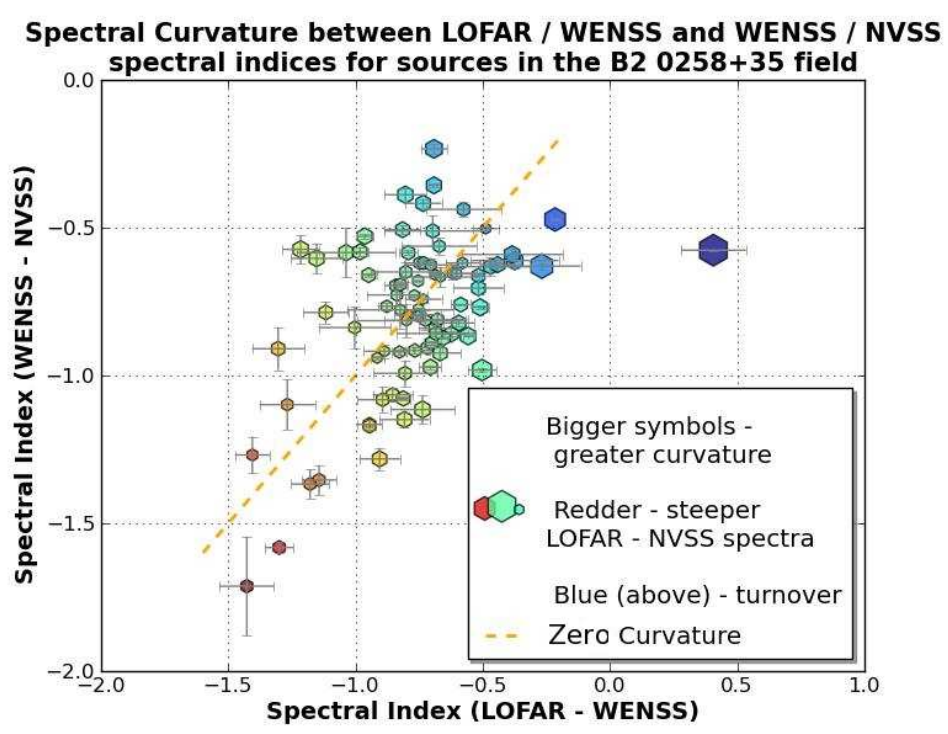

Figure 4: Curvature plot (WENSS-NVSS vs. LOFAR-WENSS spectral indices for the field centered at B2 $0258+35)$.

We have detected 11 ultra steep spectrum sources (USS) with $\alpha_{61}^{1400}<-1$ (corresponding to points in the lower left region of Figure 4). They are among the USS sources reported by de Breuck et al. (2000). This is just a taste of things to come when using LOFAR to the best of its abilities for high dynamic range, wide-field imaging at low frequencies. 


\section{Conclusions}

We have shown a detection of a relic candidate around the young CSS source B2 0258+35. These type of sources will be increasingly seen in deep LOFAR observations and we can use them to investigate the low-frequency properties of relic or relic-like sources and determine source properties tying into the duty cycle of the AGN. We have also outlined how low frequency surveys can be exploited to produce valuable insights into source properties of interest.

\section{Acknowledgements}

LOFAR, the Low Frequency Array designed and constructed by ASTRON, has facilities in several countries, that are owned by various parties (each with their own funding sources), and that are collectively operated by the International LOFAR Telescope (ILT) foundation under a joint scientific policy. The results presented here are the culmination of a great deal of work done by a large number of people. We gratefully acknowledge the engineers who have designed and built the array, as well as the large group of commissioners who have taken part in LOFAR "busy weeks" where the bulk of the commissioning progress has taken place.

Comments on the manuscript by Peter Barthel are gratefully acknowledged.

\section{References}

[1] A. Shulevski, R. Morganti, T. Oosterloo, C. Struve, Recurrent radio emission and gas supply: the radio galaxy $B 20258+35, A \mathcal{E} A 545 \mathrm{~A} 91$ [astro-ph.C0/1207.4348]

[2] D. J. Saikia, M. Jamrozy, 2009, Recurrent activity in Active Galactic Nuclei, Bulletin of the Astronomical Society of India, 37, 3-4, 63

[3] A. P. Schoenmakers, A. G. de Bruyn, H. J. A. Röttgering, H. van der Laan, The Mpc-scale radio source associated with the GPS galaxy B1144+352, AEA 341 (1) 44.

[4] N. S. Kardashev, Non-stationarity of spectra of young sources of nonthermal cosmic radio emission, Astronomicheskii Zhurnal 39 (3) 393.

[5] W. J. Jaffe, G. C. Perola, Dynamical Models of Tailed Radio-Sources in Clusters of Galaxies, $A \mathcal{E} A 26$ (3) 423.

[6] M. Murgia, C. Fanti, R. Fanti, L. Gregorini, U. Klein, K. H. Mack, M. Vigotti, Synchrotron spectra and ages of compact steep spectrum radio sources, AEA 345 (3) 769.

[7] M. Murgia, P. Parma, K. H. Mack, H. R. de Ruiter, R. Fanti, F. Govoni, A. Tarchi, S. Giacintucci, M. Markevitch, Dying radio galaxies in clusters, $A \mathcal{E} A, \mathbf{5 2 6}$, A 148

[8] E. Churazov, M. Bruggen, C. R. Kaiser, H. Bohringer, W. Forman, Evolution of buoyant bubbles in M87, ApJ, 554, 261

[9] C. Struve, T. A. Oosterloo, R. Sancisi, R. Morganti, B. H. C. Emonts, Cold gas in massive early-type galaxies: the case of NGC 1167, A E A $\mathbf{5 2 3} \mathrm{A} 75$ 
[10] B. Emonts, PhD Thesis, University of Groningen, [http://irs.ub.rug.nl/ppn/297973916]

[11] M. J. McNamara, D. A. Nulsen, J. H. Croston, Hot and cold gas accretion and feedback in radio-loud active galaxies, MNRAS, 376, 4, 1849

[12] C. de Breuck, W. van Breugel, H. J. A. Röttgering, G. Miley, A sample of 699 ultra steep spectrum radio sources to find high redshift radio galaxies, AEAS, 143, 303. 